\title{
Sociotechnical systems approach to healthcare quality and patient safety
}

\author{
Pascale Carayon $^{\mathrm{a}}$ \\ ${ }^{a}$ Center for Quality and Productivity Improvement and Department of Industrial and Systems Engineering, \\ University of Wisconsin-Madison, 1550 Engineering Drive, Madison WI 53706, USA
}

\begin{abstract}
The SEIPS (Systems Engineering Initiative for Patient Safety) model of work system and patient safety is described and selected research and practical applications are presented.
\end{abstract}

Keywords: sociotechnical systems, macroergonomics, health care, patient safety, SEIPS model

\section{Introduction}

One of the first human factors and ergonomics (HFE) studies of patient safety was conducted by Chapanis and Safren $[15,30,31]$ in 1960. Using the critical incident technique, the researchers examined medication errors and found a total of 178 medication errors that were categorized as (1) wrong patient, (2) wrong dose of medication, (3) extra unordered medication, (4) omitted medication administration, (5) wrong drug, (6) wrong timing of medication administration, and (7) incorrect route. These medication errors were caused by a range of work system factors such as failure to follow required checking procedures and verbal or written communication problems. Even though this study identified many medication errors and the work system factors that contributed to the errors, it was not until the publication of the IOM report on "To Err is Human: Building a Safer Health System" in 1999 [26] that patient safety (and human factors) received attention from healthcare organizations, policy makers, regulatory agencies and the research community.

Many healthcare professionals, experts and organizations have turned to the HFE discipline to learn HFE concepts, theories, models, tools and methods, to redesign healthcare systems and processes and to improve patient safety and quality of care [28]. Given the complexity of health care [2], HFE interventions that do not consider organizational issues are unlikely to have significant, sustainable impact on patient safety and quality of care. For instance, improving the physical design of a medical device or the cognitive interface of health information technology is important; but without understanding the organizational context in which these technologies are used, workers may develop work-arounds and the tools may not be used safely. Therefore, macroergonomics should play a major role in health care and patient safety.

We have proposed a sociotechnical systems approach to address patient safety and other quality of care problems (see Figure 1). The SEIPS (Systems Engineering Initiative for Patient Safety) model of work system and patient safety [9] is based on the macroergonomic work system model developed by Smith and Carayon [1, 12, 36, 37], and incorporates the Structure-Process-Outcome model of healthcare quality [18]. The Structure-Process-Outcome of Donabedian [18] is the most well-known model of health care quality. The integration of our macroergonomic work system model with this well-known model of healthcare quality increases the acceptability of the SEIPS model. We first describe the SEIPS model of work system and patient safety, and then its research and practical applications. 


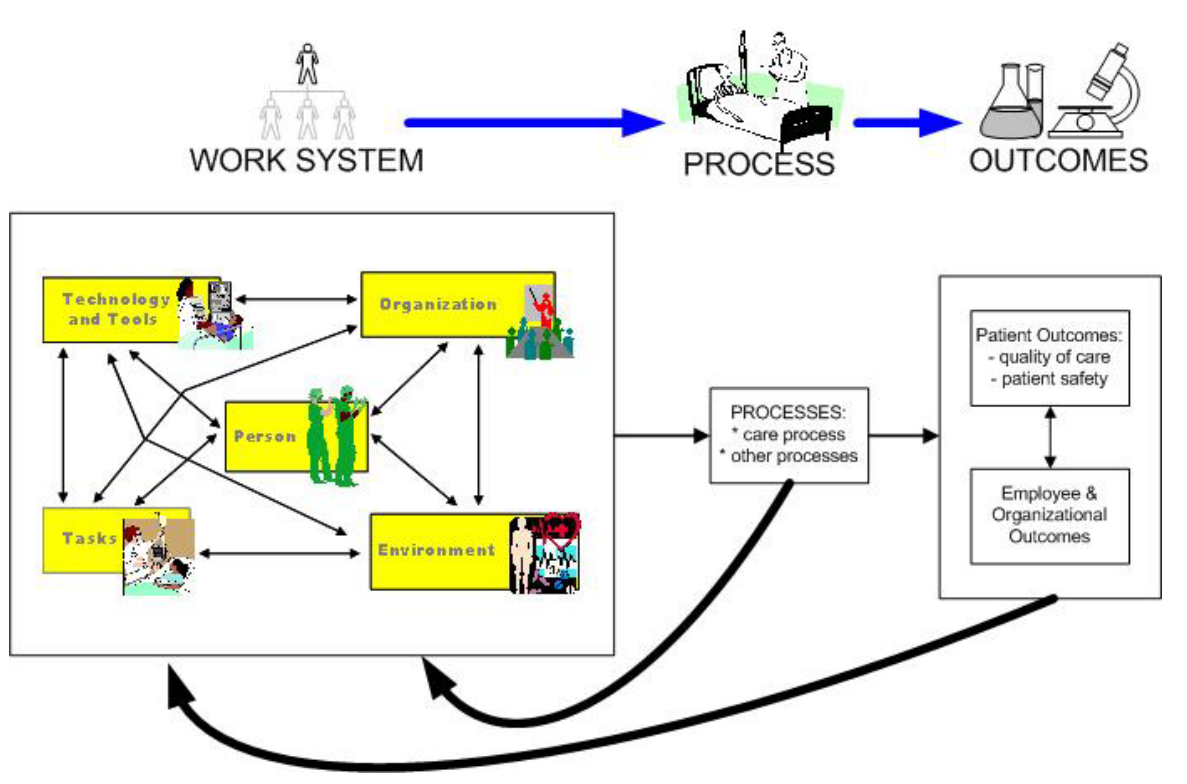

Fig. 1 - The SEIPS Model of Work System and Patient Safety [9]

\section{SEIPS model of work system and patient safety}

Key characteristics of the SEIPS model include: (1) description of the work system elements, (2) incorporation of the well-known quality of care model developed by Donabedian [18], (3) care process as being influenced by the work system and contributing to outcomes, (4) integration of patient outcomes and organizational/employee outcomes, and (5) feedback loops between the process and outcomes, and the work system (see Figure 1).

The SEIPS model of work system and patient safety represents a major tool to introduce and promote HFE in health care; it has been used by numerous healthcare researchers, professionals and educators. Because the SEIPS model integrates the macroergonomic work system model, it allows the various domains of HFE (cognitive, physical and organizational ergonomics) to be integrated and combined. This systems approach is necessary to understand the complex patient safety issues.

The SEIPS model is the conceptual framework for a range of HFE tools and methods used to evaluate healthcare work systems and processes and their impact on healthcare quality and patient safety, such as questionnaire survey $[4,23,24]$, observation methodology $[13,14]$, interviews $[3,20]$, and proactive risk assessment of healthcare processes $[8,38,40]$.

\section{Research applications of the SEIPS model of work system and patient safety}

The SEIPS model has been used by numerous healthcare researchers, professionals and educators. Researchers have used the SEIPS model to study timeliness of follow-up of abnormal test results in outpatient settings [34], to examine the safety of EHR technology [35], to evaluate ways of improving electronic communication and alerts [25], and to assess work system barriers and facilitators to the provision of outpatient pharmacy services [17]. The SEIPS model has been adopted by patient safety leaders, such as Peter Pronovost from Johns Hopkins University [29].

We have used the SEIPS model in our own research to examine patient safety in multiple care settings (e.g., ICU, pediatrics, primary care, outpatient surgery, cardiac surgery, transitions of care) and to study the implementation of various forms of health information technology (e.g., EHR, CPOE, bar coding medication administration, smart infusion pump, tele-ICU).

\subsection{Work system performance obstacles and facilita- tors}

We have used the SEIPS model to identify work system performance obstacles and facilitators of ICU 
nurses [20-22]. This research uses mixed research methods, including interviews of 15 ICU nurses [20] and a survey of 272 nurses in 17 ICUs [21, 22]. Our results show that performance obstacles can be associated with all five elements of the work system model [22]. The survey data analysis shows that obstacles related to the physical environment, familyrelated issues, supply chain management (e.g., access to supplies, stock in patient rooms, access to patient chart, delay in getting medications), and equipmentrelated issues affect ICU nurses' perception of quality and safety of care either directly or indirectly via their influence on workload [21]. These results are important as they provide information about the ICU nurses' work system factors that need to be addressed in order to improve their quality of working life as well as the quality and safety of care they provide to patients.

In a recent study, we examined performance obstacles and facilitators experienced by care managers who coordinate care for patients with chronic diseases and post-surgical patients during transitions from the hospital [5]. This study focused on care coordinators' use of multiple health IT applications, and identified performance obstacles in all of the work system elements.

\subsection{Work system impact on care processes}

A key element of the SEIPS model is the focus on care processes that may be affected by the work system. The literature on healthcare quality focuses on care processes and patient outcomes, and has not paid sufficient attention to the structural factors (work system) that can influence care processes and patient outcomes. Therefore, our research can provide useful information on how to redesign work systems in order to improve care processes and patient outcomes. Our research has examined the following care processes: outpatient surgery information management $[3,11,33]$, ICU nursing medication management [19], and the bedside rounds process in a pediatric hospital [7].

\subsection{Impact of health IT on work system and patient safety}

In many countries, health information technology (IT) is seen as a major solution to improve healthcare quality and patient safety. However, the evidence for the quality and safety benefits of health IT is limited [16]. A reason for the limited success of health IT, the failures to implement and sustain health IT, and safety problems linked to health IT is the lack of attention to HFE in the design, implementation and use of health IT. Our research has examined the impact of health IT on work system and patient safety in a variety of domains: bar coding medication administration technology in hospital settings [14, 27], smart infusion pump technology in a hospital $[10,32,39]$, and CPOE/EHR in ICUs [6, 23, 24, 41].

\section{Practical applications of the SEIPS model of work system and patient safety}

The SEIPS model can be used by healthcare organizations (1) to analyze patient safety events (e.g., root cause analysis), (2) to analyze high-risk care processes, and (3) to anticipate the potential safety consequences of sociotechnical changes such as the introduction of a new medical device or EHR technology.

Root cause analyses (RCA) are conducted to investigate sentinel events. The RCA team can use the work system model in order to systematically consider all possible work system factors that could have contributed to the sentinel event. The five elements of the work system model can be a guide when asking questions about the event: who was involved [Person]; what were they doing [Tasks]; what tools/technologies were they using [Tools/technologies]; where did the event take place [Environment]; what organizational conditions contributed to the event [Organization]. Once all of the system factors that contributed to the event have been identified, the sequence of events can be mapped in the form of a process that highlights the various system factors. We have conducted this type of analysis for a fictitious case of wrong site surgery [11].

A particular care process (or other process) may be identified as a high-risk process that probably needs redesign. The SEIPS model can be used to conduct a risk analysis to ensure that all factors contributing to the vulnerabilities or failures in the process are systematically assessed. We have conducted various types of proactive risk assessment of IV medication administration [40], ICU nursing medication management [19] and patient transfer from surgery to the ICU [8]. Proactive risk assessment methods can be used to evaluate risks in current processes as well as to evaluate risks that (could) occur with modifications in care processes associated with sociotechnical changes. 


\section{Conclusion}

The SEIPS model has become a significant "tool" for introducing and promoting HFE to healthcare researchers, professionals and educators. Knowledge of specific HFE issues (e.g., teamwork, usability, coordination, physical stressors, resilience) is necessary to study specific healthcare quality and patient safety issues. We advocate that the specialized HFE knowledge can have significant impact if it takes into account the sociotechnical system under consideration. By not understanding the broad work system, this specialized HFE knowledge is at risk of either examining the wrong problem or using the wrong approach to solve the problem. We encourage HFE researchers and practitioners to embrace the proposed sociotechnical systems approach to increase the relevance and significance of their effort targeted at improving healthcare quality and patient safety.

\section{Acknowledgments}

This publication was partially supported by grant 1UL1RR025011 from the Clinical \& Translational Science Award (CTSA) program of the National Center for Research Resources National Institutes of Health [PI: M. Drezner].

\section{References}

[1] P. Carayon, The Balance Theory and the work system model... Twenty years later, International Journal of HumanComputer Interaction 25 (2009), 313-327.

[2] P. Carayon, Human factors of complex sociotechnical systems, Applied Ergonomics 37 (2006), 525-535.

[3] P. Carayon, C.J. Alvarado, A.S. Hundt, S. Springman, and P. Ayoub, Patient safety in outpatient surgery: The viewpoint of the healthcare providers, Ergonomics 49 (2006), 470-485.

[4] P. Carayon, C.J. Alvarado, A.S. Hundt, S.R. Springman, A. Borgsdorf, and P.L.T. Hoonakker, Employee questionnaire survey for assessing patient safety in outpatient surgery, in: Advances in Patient Safety: From Research to Implementation. vol. 4, K. Henriksen, et al., Eds., ed, Agency for Healthcare Research and Quality, Rockville, MD, 2005, pp. 461-473.

[5] P. Carayon, B. Alyousef, P. Hoonakker, A.S. Hundt, R. Cartmill, J. Tomcavage, A. Hassol, K. Chaundy, S. Larson, J. Younkin, and J. Walker, Challenges to care coordination posed by the use of multiple health IT applications, in: Proceedings of the 2011 Triennial Congress of the International Ergonomics Association, ed, 2012.

[6] P. Carayon, R. Cartmill, M.A. Blosky, R. Brown, M. Hackenberg, P. Hoonakker, A.S. Hundt, E. Norfolk, T. Wetterneck, and J. Walker, ICU nurses' acceptance of electronic health records, Journal of the American Medical Informatics Association (2011).

[7] P. Carayon, L.L. Dubenske, B.C. Mccabe, B. Shaw, M.E. Gaines, M.M. Kelly, J. Orne, and E.D. Cox, Eds., Work system barriers and facilitators to family engagement in rounds in a pediatric hospital (Proceedings of the HEPS'2011 Conference, 2011, p.^pp. Pages.

[8] P. Carayon, H. Faye, A.S. Hundt, B.-T. Karsh, and T. Wetterneck, Patient safety and proactive risk assessment, in: Handbook of Healthcare Delivery Systems, Y. Yuehwern, Ed., ed, Taylor \& Francis, Boca Raton, FL, 2011, pp. 12-11.

[9] P. Carayon, A.S. Hundt, B.-T. Karsh, A.P. Gurses, C.J. Alvarado, M. Smith, and P.F. Brennan, Work system design for patient safety: The SEIPS model Quality \& Safety in Health Care 15 (2006), i50-i58.

[10] P. Carayon, A.S. Hundt, and T.B. Wetterneck, Nurses' acceptance of Smart IV pump technology, International Journal of Medical Informatics 79 (2010), 401-411.

[11] P. Carayon, K. Schultz, and A.S. Hundt, Righting wrong site surgery, Joint Commission Journal on Quality and Safety 30 (2004), 405-410.

[12] P. Carayon and M.J. Smith, Work organization and ergonomics, Applied Ergonomics 31 (2000), 649-662.

[13] P. Carayon, T.B. Wetterneck, A.S. Hundt, M. Ozkaynac, P. Ram, J. Desilvey, B. Hicks, T.L. Roberts, M. Enloe, R. Sheth, and S. Sobande, Observing nurse interaction with infusion pump technologies, in: Advances in Patient Safety: From Research to Implementation. vol. 2, K. Henriksen, et al., Eds., ed, Agency for Healthcare Research and Quality, Rockville, MD, 2005, pp. 349-364.

[14] P. Carayon, T.B. Wetterneck, A.S. Hundt, M. Ozkaynak, J. Desilvey, B. Ludwig, P. Ram, and S.S. Rough, Evaluation of nurse interaction with bar code medication administration technology in the work environment, Journal of Patient Safety 3 (2007), 34-42.

[15] A. Chapanis and M.A. Safrin, Of misses and medicines, Journal of Chronic Diseases 12 (1960), 403-408.

[16] B. Chaudhry, J. Wang, S. Wu, M. Maglione, W. Mojica, E. Roth, S.C. Morton, and P.G. Shekelle, Systematic review: Impact of health information technology on quality, efficiency, and costs of medical care, Annals of Internal Medicine 144 (2006), E12-E22.

[17] M.A. Chui, D.A. Mott, and L. Maxwell, A qualitative assessment of a community pharmacy cognitive pharmaceutical services program, using a work system approach, Research in Social and Administrative Pharmacy (2011).

[18] A. Donabedian, The quality of medical care, Science 200 (1978), 856-864

[19] H. Faye, A.J. Rivera-Rodriguez, B.T. Karsh, A.S. Hundt, C. Baker, and P. Carayon, Involving intensive care unit nurses in a proactive risk assessment of the medication management process, The Joint Commission Journal on Quality and Patient Safety 36 (2010), 376-384.

[20] A. Gurses and P. Carayon, A qualitative study of performance obstacles and facilitators among ICU nurses, Applied Ergonomics 40 (2009), 509-518.

[21] A. Gurses, P. Carayon, and M. Wall, Impact of performance obstacles on intensive care nurses workload, perceived quality and safety of care, and quality of working life, Health Services Research (2009), 422-443.

[22] A.P. Gurses and P. Carayon, Performance obstacles of intensive care nurses, Nursing Research 56 (2007), 185-194.

[23] P. Hoonakker, P. Carayon, and J.M. Walker, Measurement of CPOE end-user satisfaction among ICU physicians and nurses, Applied Clinical Informatics 1 (2010), 268-285. 
[24] P.L.T. Hoonakker, R.S. Cartmill, P. Carayon, and J.M. Walker, Development and psychometric qualities of the SEIPS survey to evaluate CPOE/EHR implementation in ICUs, International Journal of Healthcare Information Systems and Informatics 6 (2011), 51-69.

[25] S. Hysong, M. Sawhney, L. Wilson, D. Sittig, A. Esquivel, M. Watford, T. Davis, D. Espadas, and H. Singh, Improving outpatient safety through effective electronic communication: a study protocol, Implementation Science 4 (2009), 62.

[26] L.T. Kohn, J.M. Corrigan, and M.S. Donaldson, Eds., To Err is Human: Building a Safer Health System, National Academy Press., Washington, D.C., 1999, p.`pp. Pages.

[27] R. Koppel, T. Wetterneck, J.L. Telles, and B.-T. Karsh, Workarounds to barcode medication administration systems: Their occurrences, causes, and threats to patient safety, Journal of the American Medical Informatics Association 15 (2008), 408-423.

[28] L.L. Leape, D.M. Berwick, and D.W. Bates, What practices will most improve safety? Evidence-based medicine meets patient safety, Journal of the American Medical Association 288 (2002), 501-507.

[29] P.J. Pronovost, C.A. Goeschel, J.A. Marsteller, J.B. Sexton, J.C. Pham, and S.M. Berenholtz, Framework for patient safety research and improvement, Circulation 119 (2009), 330337.

[30] M.A. Safren and A. Chapanis, A critical incident study of hospital medication errors - Part 1, Hospitals 34 (1960), 3234;57-66.

[31] M.A. Safren and A. Chapanis, A critical incident study of hospital medication errors - Part 2, Hospitals 34 (1960).

[32] M.E. Schroeder, R.L. Wolman, T.B. Wetterneck, and P. Carayon, Tubing misload allows free flow event with Smart intravenous infusion pump, Anesthesiology 105 (2006), 434 435.

[33] K. Schultz, P. Carayon, A.S. Hundt, and S. Springman, Care transitions in the outpatient surgery preoperative process: Fa- cilitators and obstacles to information flow and their consequences, Cognition, Technology \& Work 9 (2007), 219-231.

[34] H. Singh, E.J. Thomas, S. Mani, D. Sittig, H. Arora, D. Espadas, M.M. Khan, and L.A. Petersen, Timely follow-up of abnormal diagnostic imaging test results in an outpatient setting: Are electronic medical records achieving their potential?, Archives of Internal Medicine 169 (2009), 1578-1586.

[35] D.F. Sittig and H. Singh, Eight rights of safe electronic health record use, Journal of the American Medical Association 302 (2009), 1111-1113

[36] M.J. Smith and P. Carayon-Sainfort, A balance theory of job design for stress reduction, International Journal of Industrial Ergonomics 4 (1989), 67-79.

[37] M.J. Smith and P. Carayon, Balance theory of job design, in: International Encyclopedia of Ergonomics and Human Factors, W. Karwowski, Ed., ed, Taylor \& Francis, London, 2000, pp. 1181-1184.

[38] T.B. Wetterneck, A.S. Hundt, and P. Carayon, FMEA team performance in health care: A qualitative analysis of team member perceptions, Journal of Patient Safety 5 (2009), 102108 .

[39] T.B. Wetterneck, K.A. Skibinski, T.L. Roberts, S.M. Kleppin, M. Schroeder, M. Enloe, S.S. Rough, A.S. Hundt, and P. Carayon, Using failure mode and effects analysis to plan implementation of Smart intravenous pump technology, American Journal of Health-System Pharmacy 63 (2006), 15281538 .

[40] T.B. Wetterneck, K.A. Skibinski, T.L. Roberts, S.M. Kleppin, M.E. Schroeder, M. Enloe, S.S. Rough, A.S. Hundt, and P. Carayon, Using failure mode and effects analysis to plan implementation of smart i.v. pump technology, American Journal of Health-System Pharmacy 63 (2006), 1528-1538.

[41] T.B. Wetterneck, J.M. Walker, M.A. Blosky, R.S. Cartmill, P. Hoonakker, M.A. Johson, E. Norfolk, and P. Carayon, Factors contributing to an increase in duplicate medication order errors after CPOE implementation, Journal of the American Medical Informatics Association (2011). 